\title{
Challenges for the Wendelstein 7-X Magnet Systems during the next Operation Phase
}

\author{
Thomas Rummel, Konrad Risse, Michael Nagel, Thomas Mönnich, Frank Füllenbach, Hans-Stephan \\ Bosch, and the W7-X Team
}

\begin{abstract}
During the first operation phase OP1.1 of Wendelstein 7-X (W7-X) the magnet systems were not operated up to its maximum capabilities. During the next operation phase OP1.2 a big step in the direction to a full current operation will be taken.

The superconducting magnet system consists of the two different coil types: the non planar coils (NPC) and the planar coils (PLC). With respect to OP1.1 the NPC current in OP1.2 will be increased slightly, but will be doubled in the PLC. Also a reversal of the current direction in the PLC will be required. Tests during and after OP1.1 showed that it might be advantageous to reduce the electrical stress during fast discharges. Therefore the magnet protection system was optimized. In order to avoid the risk of a quench the magnet system is being operated with a certain temperature margin with respect to the critical temperature of the superconductor. The safety operation system will be updated to secure automatic observation and reaction.

The five trim coils are normal conducting coils mounted at the outer surface of the cryostat. They were operated during OP1.1 up to $2 / 3$ of the maximum current. Therefore full current operation needs to be tested for the first time. For OP1.2 also measures were studied and installed to minimize the cross link between the trim coils and the superconducting main field coils.
\end{abstract}

Index Terms - Operating systems, Stellarators, Superconducting magnets, Wendelstein 7-X.

\section{INTRODUCTION}

$\mathrm{T}$ HE fusion device Wendelstein 7-X (W7-X) went into operation in 2015 and completed the first plasma operation phase OP1.1 successfully in 2016 [1,2]. The aim of OP1.1 was to check and to operate all relevant systems and to generate first helium and hydrogen plasmas. The behavior of the superconducting magnet system meets in general all the expectations. It was possible to run the system stable up to eight hours at $13.47 \mathrm{kA}$ which represents $74 \%$ of the finally expected highest current. The superconducting magnets were energized 35 times on 30 operation days for about 183 hours to secure the first plasma operation phase. The high availability of $94 \%$ of the superconducting magnet system, which includes cryogenic and power supply, as well as quench detection system during the plasma operation is another important result. Only two out of the planned 32 operation days had to be skipped due to the unavailability of the magnet system. No quenches occurred; the electrical, the thermal as well as the mechanical behavior are a good basis for the operation at higher currents and other, more demanding magnetic configurations [3]. Nevertheless most of the W7-X systems were not operated up to the full capabilities. Also the magnet systems were not operated up to the maximum currents.

During the next operation phase OP1.2 an important step in the direction to a full performance plasma operation will be taken. The detailed program for the plasma physics research will be developed during this 3 months plasma operation period. Therefore a detailed commissioning program for the magnet systems cannot be set up beforehand. Nevertheless general requirements like higher currents and voltages are already know and studies, calculations and even changes of components have been performed in preparation of this next phase. The paper describes the new requirements and the supporting measures in preparation of the commission for the next operation phase OP1.2.

\section{SUPERCONDUCTING MAGNET SYSTEM}

\section{A. Layout}

The superconducting magnet system consists of two main coil types, the 50 non planar coils generate the main magnetic stellarator field, whereas the 20 planar coils generate additional fields to provide the experimental flexibility of the device. The non planar coils have dimensions of about $3.5 \times 2.5 \times 1.5$ meters and a weight of about 6 tons, whereas the planar coils are more circular with diameter of about 4 meters. Their weight is about 3 tons per coil.

Financial support acknowledgment. "This work has been carried out within the framework of the EUROfusion Consortium and has received funding from the Euratom research and training programme 2014-2018 under grant agreement No 633053. The views and opinions expressed herein do not necessarily reflect those of the European Commission.”

All authors are with the Max-Planck-Institute for Plasma Physics, D-17491 Greifswald, Germany (e-mail: thomas.rummel@ ipp.mpg.de). 
Ten coils of the same type are forming one electrical circuit. Consequently there are 5 circuits containing the non planar coils (NPC1, .., NPC5) and two circuits containing the planar coils (PLC A and PLC B). The circuits represent a load of up to $1 \mathrm{H}$ each and are individually powered with currents of up to $18.2 \mathrm{kA}$ in maximum. The experimental flexibility of W7-X is achieved by variation of the relation between the current amplitude in the different non planar coil circuits, the polarity of the planar coil currents and combinations of both features. This allows modifying the magnetic field in terms of horizontal position, shear, rotation transformation iota and mirror. Fig. 1 shows the positions of the coil types within one of five equal magnet modules in W7-X. Along one module the plasma shape changes from a bean shape to a triangular shape near the type 5 coils back to the triangular shape near the type one coil. The neighboring modules left and right start with a coil 1 type.

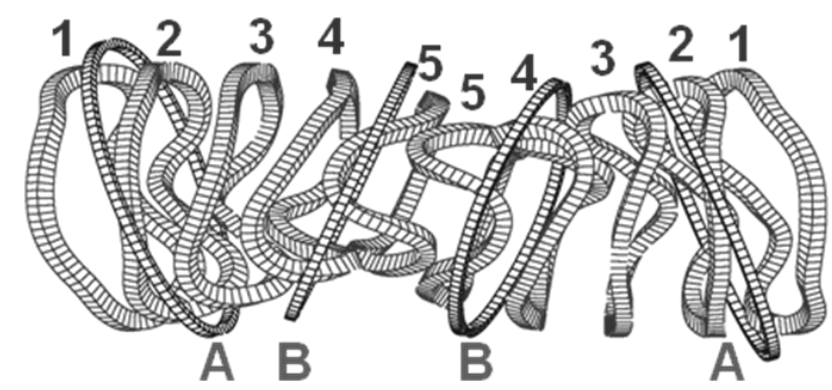

Fig. 1. One of the five magnet modules of W7-X showing the non planar coil types 1 to 5 and the planar coil types A and B.

\section{B. Operation field of $W 7-X$}

Fig. 2 shows the possible operation field of W7-X and basic settings for the current in the non planar and planar coil circuits.

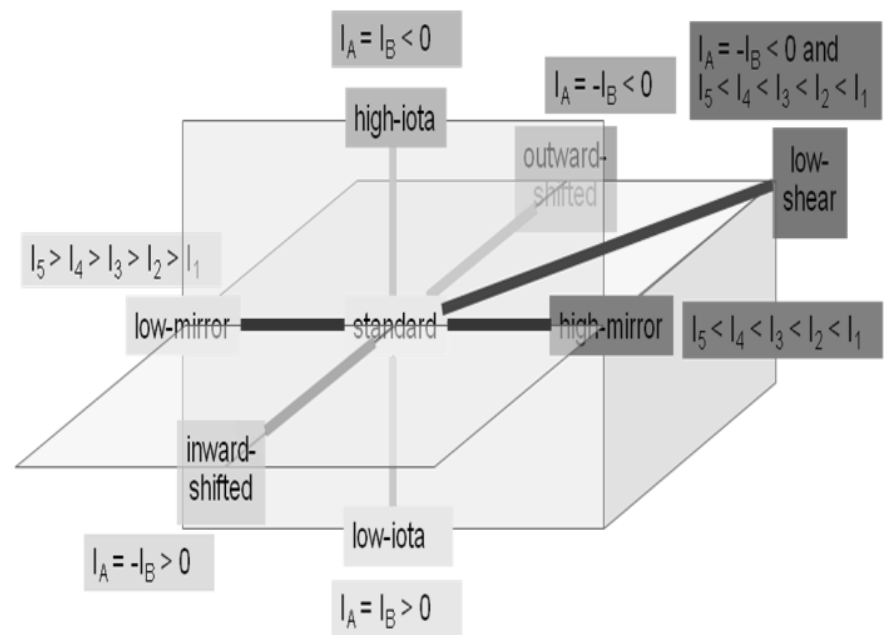

Fig. 2. Possible operation field of the W7-X superconducting magnet system and principle coil currents in the non planar $\left(\mathrm{I}_{1}\right.$ to $\left.\mathrm{I}_{5}\right)$ and the planar coils $\left(\mathrm{I}_{\mathrm{A}}\right.$ and $\left.\mathrm{I}_{\mathrm{B}}\right)$. Picture: Joachim Geiger.

The so called "standard" configuration is achieved if all the coil currents in the non-planar coils are equal and the currents in the planar coils are zero. As indicated in Fig. 2 the magnetic configuration and the plasma can be varied on several surfaces. One surface is formed by the horizontal lines of high iota and low iota and the vertical lines of low mirror and high mirror. The total area within the rectangle is reachable by variation of non planar coil current amplitudes and planar coil currents amplitude and direction. As one example one can reach the high iota configuration, by having equal non planar coil currents in the five circuits and have the coil currents in the planar coils PLCA and PLCB equal to each other and in the same direction as of the non planar coils. The change of the magnetic mirror will be achieved by the relation of the current amplitude among the five non planar coil circuits. If the currents in the NPC5 coils are the highest and in the NPC1 the lowest one reaches a low mirror configuration. If the currents are the highest in the NPC1 coils one reaches the high mirror configuration. A similar surface in the operation field than described above can be created by the borderlines of low and high mirror and inward and outward shift.

A more challenging configuration, also for the control of the coil currents, will be the combination of different coil currents among the five non planar circuits and the changed polarity of one of the planar coil circuits (e.g. "low shear" in Fig. 2).

To have the full overview about the flexibility of W7-X it might be important to know that the absolute value of the currents can also vary to operate $\mathrm{W} 7-\mathrm{X}$ at $2.5 \mathrm{~T}$ or at $3 \mathrm{~T}$ on the magnetic axis. But also other basic fields are possible, based on the 
resonance frequencies of the electron cyclotron resonance heating systems.

\section{C. $\quad$ Increased coil currents in OP1.2}

In OP1.1 only two of the nine predefined magnetic configurations have been used ("Standard" and a configuration half the way to "low-iota"; see Fig. 2").

In terms of electrical currents the non planar coils have been operated up to $13.47 \mathrm{kA}$, and the planar coils up to $5 \mathrm{kA}$, respectively. During the next phase the coil currents have to be increased to $15.3 \mathrm{kA}$ in the non-planar coils and to $10.1 \mathrm{kA}$ in positive direction and up to $-10.3 \mathrm{kA}$ in opposite direction in the planar coils, respectively.

Table 1 shows the full set of currents for the foreseen magnetic configurations. The new maximum values for operations are encircled. In OP1.1 only the configurations "A" and "J" have been used.

TABLE I

CURRENTS IN THE NON-PLANAR ( $\mathrm{I}_{1}$ TO $\mathrm{I}_{5}$ ) AND IN THE PLANAR COILS $\left(\mathrm{I}_{\mathrm{A}}, \mathrm{I}_{\mathrm{B}}\right)$ FOR THE MAGNETIC CONFIGURATIONS

\begin{tabular}{llcccccccc}
\hline \multirow{2}{*}{ Configuration } & $\begin{array}{c}\mathbf{B}_{0} \\
{[\mathrm{~T}]}\end{array}$ & $\begin{array}{c}\mathrm{I}_{1} \\
{[\mathrm{kA}]}\end{array}$ & $\begin{array}{c}\mathrm{I}_{2} \\
{[\mathrm{kA}]}\end{array}$ & $\begin{array}{c}\mathrm{I}_{3} \\
{[\mathrm{kA}]}\end{array}$ & $\begin{array}{c}\mathrm{I}_{4} \\
{[\mathrm{kA}]}\end{array}$ & $\begin{array}{c}\mathrm{I}_{5} \\
{[\mathrm{kA}]}\end{array}$ & $\begin{array}{c}\mathrm{I}_{\mathrm{A}} \\
{[\mathrm{kA}]}\end{array}$ & $\begin{array}{c}\mathrm{I}_{\mathrm{B}} \\
{[\mathrm{kA}]}\end{array}$ \\
\hline A & Standard & 2.5 & 13.5 & 13.5 & 13.5 & 13.5 & 13.5 & 0.0 & 0.0 \\
B & Low iota & 2.5 & 12.2 & 12.2 & 12.2 & 12.2 & 12.2 & 9.2 & 9.2 \\
C & High iota & 2.5 & 14.9 & 14.9 & 14.9 & 14.9 & 14.9 & -10.3 & -10.3 \\
D & Low mirror & 2.5 & 12.6 & 13.2 & 13.2 & 14.2 & 14.2 & 0.0 & 0.0 \\
E & High mirror & 2.5 & 14.5 & 14.1 & 13.4 & 12.8 & 12.4 & 0.0 & 0.0 \\
F & Low shear & 2.5 & 15.3 & 15.0 & 14.2 & 11.5 & 11.4 & -9.8 & $\underbrace{}_{10.2}$ \\
G & Inward shift & 2.5 & 13.1 & 12.9 & 13.2 & 14.6 & 14.7 & 4.1 & -8.2 \\
H & Outward shift & 2.5 & 14.0 & 14.0 & 13.6 & 13.0 & 13.0 & -5.7 & 5.7 \\
I & Limiter & 2.5 & 14.2 & 14.6 & 13.5 & 12.2 & 11.8 & -4.0 & 7.9 \\
J & Limiter OP1.1 & 2.5 & 12.8 & 12.8 & 12.8 & 12.8 & 12.8 & 5.0 & 5.0 \\
\hline
\end{tabular}

Doubling the current in the planar coils is an especially big step for the system. It affects the electrical, the thermo hydraulic and the mechanical behavior. Also a reversal of the current direction in the PLC will be required. That means that the current in one or two planar coil circuits will run in the opposite direction than in the non planar coil circuits. Due to the magnetic coupling between all systems the controller of each power supply will be affected by the other systems.

\section{Optimized dump resistor}

In OP1.1 the maximum voltage during a fast discharge was $2.1 \mathrm{kV}$. For OP1.2 a maximum voltage of up to $2.7 \mathrm{kV}$ is expected, based on simulations.

Tests during and after OP1.1 showed that it might be advantageous to reduce the electrical stress during fast discharges. The combination of both, the expected higher voltage in OP1.2 and the necessity to come down with the voltage, forced the decision to study possible reduction measures and to integrate necessary changes on the magnet protection system. Therefore the magnet protection system was reviewed and an optimized dump resistor was proposed. It is customized for the next operation phase only, but reduces the voltage during a fast discharge significantly from $2.7 \mathrm{kV}$ to $1.8 \mathrm{kV}$. Due to the limited time between the two operation phases of less than a year, no new dump resistors could be designed, ordered, manufactured, tested and assembled. As a way out, it was studied to use the existing dump resistors and change them. One dump resistor has a resistance of $148 \mathrm{mOhm}$ and is composed of the series connection of six equal parts with a resistance of $24.7 \mathrm{mOhm}$ each. After studies and simulation runs a combination of series and parallel connection of the six parts have been found, which reduces the resistance significantly to $74 \mathrm{mOhm}$, but nearly keep the capability to absorb the stored energy coming from the coils. Special attention had to be given to the heat development of the resistor. The resistor material is highly temperature dependant, because pure nickel is used. In a series connection of all six parts the heat development is uniform and balanced. In a combination of series and parallel connection the part in series will be loaded with much more energy and consequently will have a higher temperature rise. At the end of the fast discharge the new resistor will have a maximum temperature of simulated 600 degrees Celsius compared to 270 degrees Celsius of the old dump resistor. The maximum allowed temperature is $650{ }^{\circ} \mathrm{C}$. It is obvious that the new resistor is designed very close to the limit and does not allow higher currents i.e. higher energy in the coils. Another consequence of the changed dump resistor is the reduced dumping speed of the fast discharge. This is a positive result, because a slower change of the current and consequently a lower mechanical impact to the coils is requested from the mechanical point of view [4].

Also from the cryogenic point of view this effect is positive, the warming of the coil casing due to eddy currents is reduced and the heat input to the helium gas is reduced as well. The heat input is roughly proportional to the inverse of the time constant 
of the current decay. Therefore the helium refrigerator sees less heat load. This reduces the risk of a trip of the cold machines and will therefore increase the availability of the refrigerator.

Another positive effect can be seen in case of a fast discharge from magnetic configurations with negative coil currents in the planar coils (next chapter).

\section{E. Fast discharge from current with opposite polarities}

In case of a quench or another severe impact to the superconducting coils, a fast discharge will be necessary. The new challenge in OP1.2 will be that two coil groups can have the opposite polarity of the coil current with respect to the other five groups. It has to make sure that also such situation must not lead to unacceptable results. Fig. 3 shows the currents in the non planar as well as in the planar coils after a fast discharge from the high iota configuration using the old dump resistor.

Before the start of the fast discharge the currents in the five non planar coil circuits are positive at $14.88 \mathrm{kA}$ and the currents in the planar coils are negative with $-10.3 \mathrm{kA}$. During the fast discharge the effect of the magnetic coupling between the systems and the rule of flux conservation play an important role to explain the current trace of the planar coils. Due to the flux conservation and the much lower inductance the current in the planar coils decreases fast, and then crosses zero and flows in the same direction as in the non planar coils. The result is a jump in the planar coil current from $-10.3 \mathrm{kA}$ to $+8 \mathrm{kA}$ within a few seconds (at $4.5 \mathrm{sec}$. absolute in Fig. 3). Mechanical stress, warming due to eddy currents produces a certain load onto the coils, the magnet support structure or to the cryogenic system which has to handle the higher temperatures.

Also these issues will be less critical by slowing down the fast discharge speed. The current jump is smaller and slower and reaches $+6 \mathrm{kA}$ only at $6 \mathrm{sec}$. absolute, also the thermal influence is therefore more relaxed.

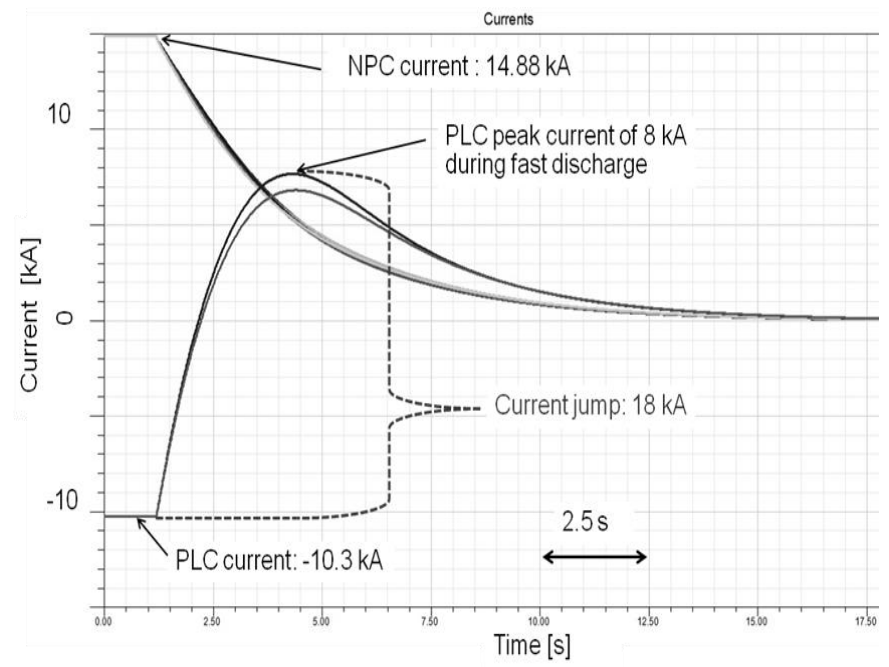

Fig. 3. Currents in the non-planar and the planar coils before and during a fast discharge (old dump resistor).

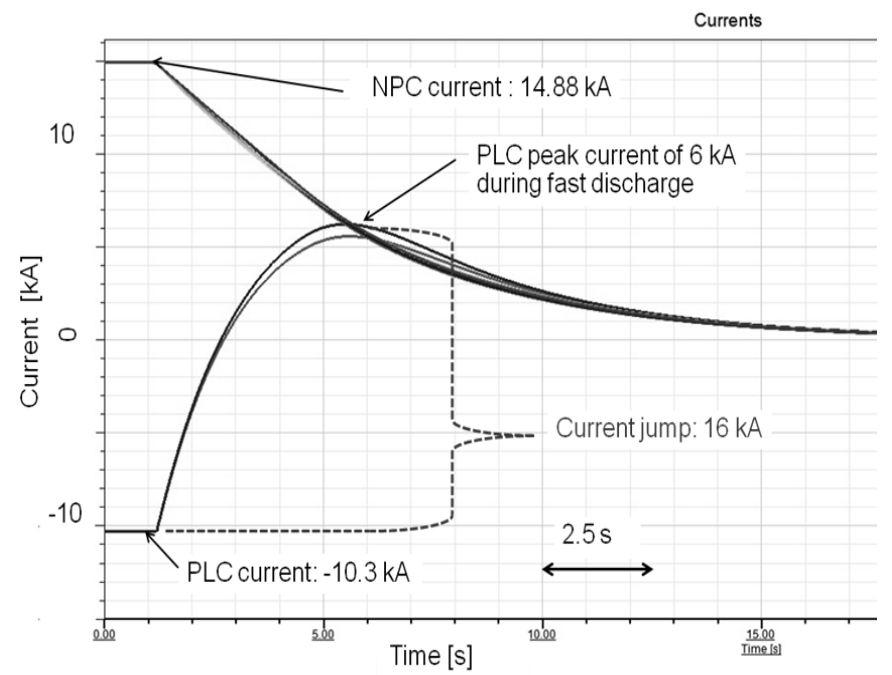

Fig. 4. Currents in the non-planar and the planar coils before and during a fast discharge (modified dump resistor). 
The simulations have shown that the system is prepared for this new kind of fast discharges.

\section{F. Hot Spot temperature of the superconductor}

Unfortunately the reduced dumping speed has also a negative consequence. A slower ramp down of the current in case of a quench increases the hot-spot-temperature in the superconductor after a quench. Therefore the studies to reduce the voltage by changing the dump resistor were accompanied by studies about the hot spot temperature evolution after a quench. Due to the importance of the hot spot temperature for the integrity of the superconducting magnet system the studies were performed inhouse and in parallel by external experts. As a result the hot spot temp after a quench will not exceed the specified limit of $130 \mathrm{~K}$ [6] with the new dump resistor.

It has to be reminded that higher currents than presently foreseen for OP1.2 will require new calculations and most likely again a new dump resistor.

\section{G. Handling of the cryogenic sensor signals}

In order to minimize the risk of a quench the magnet system will be operated with a temperature margin of one Kelvin or more with respect to the critical temperature of the superconductor. An automatic observation was planned which monitors the temperatures of the conductor cooling. The respective helium temperatures at inlet and outlet of the coils and at bus bar joints are measured. Then $1 \mathrm{~K}$ is added to the maximum measured temperature and the corresponding critical current is calculated. The calculation is done for the maximum magnetic field for the given configuration. This current is then compared to the actual current in the coils. In case the coil current is higher than the calculated an automatic current ramp down should be initiated.

During OP 1.1 the cryogenic system of W7-X worked well, but a number of temperature sensors at coils or bus bars showed higher temperatures than expected and did prevent to use that described protection algorithm [5]. Some of those sensors were located in regions with smaller magnetic field like bus bar joints at the current leads and could therefore tolerate higher temperatures. Other sensors showed systematic deviations compared to reference sensors and were considered malfunctioning. As in OP1.1 all conductor and bus bar sensors are automatically used to calculate the critical current, some sensors blocked the automatic safety function. The higher currents in OP1.2 will require a more reliable observation of the cryogenic behavior and a more appropriate reaction to deviations. After OP1.1 malfunctioning sensors were checked at room temperature. The resistance was checked and compared with measurements before the cool down. The proper allocation to the calibration data in the measuring module was verified and a test to check potential mix up of the wiring was done. A replacement of those temperature sensors inside the cryostat is considered very difficult and was not done so far. But the software was upgraded in a way that the operator is now able to exclude sensors from the calculation in case of malfunction also during operation. This measure allows using the automated critical current calculation as criteria for the operation of the coils and will secure the steady state operation even in case of small sensor failures.

\section{TRIM COIL SYSTEM}

\section{A. Layout}

The second coil system of W7-X is the so-called trim coil system, intended for enhanced experimental flexibility and error field corrections. It consists of five water cooled copper coils of two different types A and B, placed at the outer side of the cryostat. Four coils (type A) are identical in size and shape. They have overall dimensions of $3.5 \times 3.3$ meters with 48 turns and will be operated with currents of up to $1.8 \mathrm{kA}$. They represent a load of $19 \mathrm{mH}$ and $53 \mathrm{~m} \Omega$ each. The trim coil type B is smaller (2.8x2.2 meters) than the type A coils, but has more turns (72) and will be operated at higher currents (up to $1.95 \mathrm{kA}$ ). Its electrical parameters are $26 \mathrm{mH}$ and $29 \mathrm{~m} \Omega$ [7].

\section{B. Increased coil currents in $O P 1.2$}

The trim coils were operated during OP1.1 up to $1.1 \mathrm{kA}$ for type A and up to $1.2 \mathrm{kA}$ for type B coil, which represents $2 / 3$ of the maximum current only. In OP1.2 the full current will be required. In preparation of the new challenge the power supplies' control system, the monitoring of the coils temperature and water cooling have been strengthened.

\section{Fast discharge of the coils}

An important and necessary improvement resulted from the evaluation of one unexpected fast discharge of the superconducting magnet system. The trim coil power supplies detected an internal failure and switched off. This induced via the magnetic coupling a strong and fast voltage peak in the superconducting coils. The quench detection system interpreted this voltage as a sign of a quench and triggered a fast discharge of the superconducting system. The higher currents in OP1.2 in trim 
and superconducting coils wil make this cross link even more likely. Therefore measures were studied and installed to minimize the cross link between the two coil systems for OP1.2.

A more detailed investigation of the event discovered a very fast current ramp down of the trim coil current during its fast discharge. The ramp rate was above $-20 \mathrm{kA} / \mathrm{s}$. A simulation of the magnetic coupling between the trim coils and the superconducting coils with its six pancakes showed induced voltages in the superconducting coils which exceeds the threshold limit of the quench detectors. In principle there are two basic strategies to overcome the issue. The quench detection system could be made more insensitive against voltage in the superconducting coils. Unfortunately this also reduces the sensitivity of the quench detection process itself. Also an increase of the threshold level and / or the integration time would significantly reduce the performance of the quench detection system. Integration time is the time in which the threshold voltage level has to be above the limit in order to define the signal to be a quench.

The second strategy avoids the changes of the quench detection system. Instead it is based on changes in the trim coil power supplies. If a severe internal failure in the power supply is detected, like a damage of one of the Insulated-Gate-BipolarTransistors (IGBT), the gate signals to the IGBTs will be interrupted. This leads in the present configuration to an immediate stop of the output power and simultaneously the free wheel diode of the load circuit will be opened. Due to the small inductance and the relatively high resistance the current will decrease fast. A change in the power supply should change this behavior. It was decided to implement the second strategy because it is easier to implement and it avoids the change of the quench detection system which is treated in W7-X as an approved safety system.

\section{New fast discharge control of the power supplies}

Each power supply unit consists of a $400 \mathrm{~V}$ input grid transformer, followed by two diode six pulse bridges creating a DC bus, buffered with capacitors of $268 \mathrm{mF}$. The power conversion is being made by H-bridges using Insulated-Gate-Bipolar-Transistors (IGBT) with internal freewheeling diode. Three of these H-bridges are connected in parallel forming a subassembly. Four of these subassemblies are connected in parallel. That means internally there are 12 parallel H-bridge circuits and it will be extremely unlikely that all circuits will have a failure at the same time. The new control concept acts as following: If a failure as described above is detected, the affected H-bridge will be switched off like in the old concept, but the remaining H-bridges will ramp down the current to zero in a controlled and linear way with a defined ramp of $-2 \mathrm{kA} / \mathrm{s}$ instead of the former $-20 \mathrm{kA} / \mathrm{s}$. Fig. 5 shows the fast discharge of the current starting from the maximum current of $1800 \mathrm{~A}$ and the related voltage after the modification. The ramp of the current is now linear and the ripple of the voltage is a sign of the actively controlled ramp down.

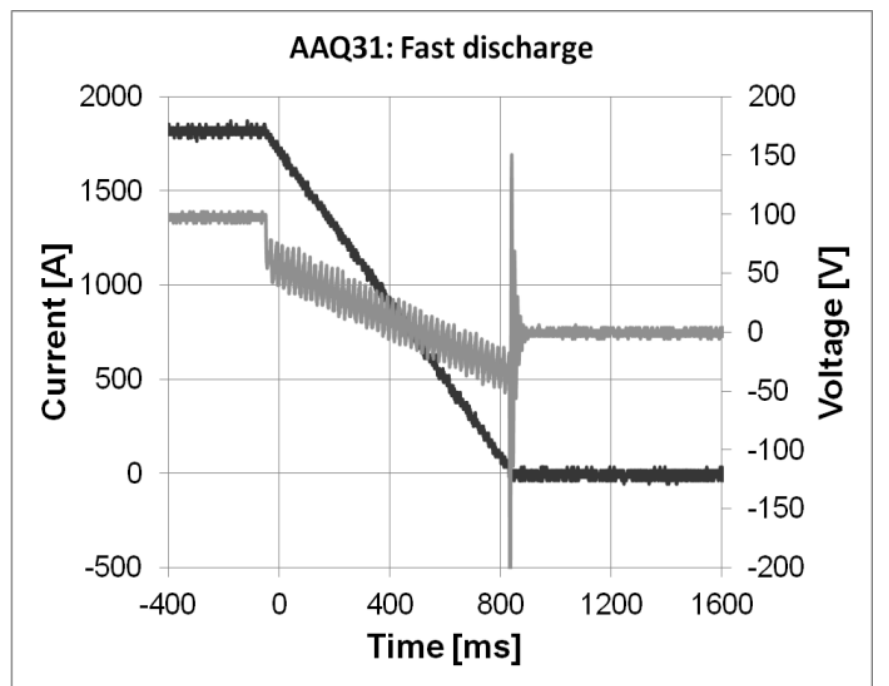

Fig. 5. Fast discharge of a trim coil after the modification of the discharge control system (current: black; voltage: grey).

This new concept has been tested first using a dummy load and finally using the trim coils. Tests with operation current in the trim coils and activated quench detection system of the superconducting coils have shown that there is no undesirable influence on the quench detection system anymore.

\section{CONCLUSION}

After the successful operation of Wendelstein 7-X during the first plasma operation phase main challenges for the magnet systems in the upcoming operation phase OP1.2 will bring the machine a big step forward in the direction of the full 
performance. The superconducting magnet system will have to show a more powerful operation with higher currents and special operation scenarios with different coil current direction in the magnetically coupled systems. Updates of the control system and modification of the dump resistors prepared the system for the new challenges.

The trim coils have been also prepared for the next operation phase which requires the operation with the maximum current. One important issue, the interference with the quench detection system of the superconducting coils has been solved by an alternative approach to modify the power supplies control system.

In conclusion the magnet systems of $\mathrm{W} 7-\mathrm{X}$ are ready for the next operation phase which is going to start at the beginning of July 2017.

\section{REFERENCES}

[1] H.-S. Bosch, V. Bykov, R. Brakel, P. van Eeten, J.-H. Feist, M. Gasparotto, H. Grote, T. Klinger, M. Nagel, D. Naujoks, M. Otte, K. Risse, T. Rummel, J. Schacht, T. Sunn Pedersen, R. Vilbrandt, L. Wegener, A. Werner, R. Wolf, the W-X Team., "Final integration, commissioning and start of the Wendelstein 7-X stellarator operation," Nuclear Fusion, vol. 57, no. 11, p. 116015, 2017.

[2] T. Sunn Pedersen et al., "Key results from the first plasma operation phase and outlook for future performance in Wendelstein 7-X," Physics of Plasmas, vol. 24, p. 055503, 2017.

[3] Th. Rummel, M. Nagel; V. Bykov; D. Birus; A. Carls; C. P. Dhard; E. Köster; Th. Mönnich; K. Riße; M. Schneider; H.-S. Bosch, „Commissioning Results of the Superconducting Magnet System of Wendelstein 7-X,“ IEEE Transactions on Applied Superconductivity, Vol. 27, No. 4, p. 4200307, June 2017.

[4] V. Bykov, A. Carls, J. Zhu, P. van Eeten, L. Wegener, H-S. Bosch and W7-X team, "Mechanical Monitoring Issues in Preparation to Next Step of Wendelstein 7-X Operation," IEEE Transactions on Plasma Science, vol. PP, issue 99, pp. 1-9.

[5] M. Nagel, C.P. Dhard, H. Bau, H.-S. Bosch, U. Meyer, S. Raatz, K. Risse, T. Rummel, "Cryogenic commissioning, cool down and first magnet operation of Wendelstein 7-X," IOP Conf. Series: Materials Science and Engineering 171 (2017) 012050, doi:10.1088/1757899X/171/1/012050.

[6] J. H. Feist, "System specification of WENDELSTEIN 7-X," internal report, Doc. No. 1-NBB-S01.0, July 1999.

[7] Th. Rummel, K. Riße, F. Füllenbach, M. Köppen, J. Kißlinger, T. Brown, R. Hatcher, St. Langish, M. Mardenfeld, and H. Neilson, „The Wendelstein 7-X Trim Coil System,“ IEEE Transactions on Applied Superconductivity, Vol. 24, No. 3, p. 4200904, June 2014. 